\title{
A brief description of the GPS architecture
}

\section{Una descripción breve de la arquitectura del GPS}

MEDINA-CASTRO, Paul $\dagger^{*}$, CARAVEO-MENA, Camilo, BARBOZA-TELLO, Norma Alicia and LOREDO-MEDINA, Raúl

Universidad Autónoma de Baja California, Faculty of Engineering Sciences and Technology, Mexico.

Instituto Tecnológico Superior de Guasave, Mexico.

ID $1^{\text {st }}$ Author: Paul, Medina-Castro / ORC ID: 0000-0003-3306-1494, CVU CONACYT ID: 42360

ID $1^{\text {st }}$ Co-author: Camilo, Caraveo-Mena / ORC ID: 0000-0001-6104-3061, CVU CONACYT ID: 405987

ID $2^{\text {nd }}$ Co-author: Norma Alicia, Barboza-Tello / ORC ID: 0000-0002-1009-3672, CVU CONACYT ID: 46396

ID $3^{\text {rd }}$ Co-author: Raúl, Loredo-Medina / ORC ID: 0000-0002-0785-5437, CVU CONACYT ID: 273827

DOI: $10.35429 / E J D R C .2020 .11 .6 .12 .16$

Received July 1, 2020; Accepted December 30, 2020

\begin{abstract}
The GPS is a radio-satellite system that allows to the GPS receiver to figure out its tridimensional position on the globe (latitude, longitude, and elevation), as well as allows it to determine its velocity and to make precise timing measurements. This article describes the architecture of the GPS system for the nonspecialized reader. The objective is that the lector has an idea of all that happens when using some equipment or application based on this technology.
\end{abstract}

GPS, GNSS, Satellites

\section{Resumen}

El sistema GPS es un sistema de radio satelital a través del cual el usuario puede determinar su posición tridimensional (latitud, longitud y altitud), su velocidad y hacer mediciones de tiempo muy precisas. En este artículo se describe la arquitectura del sistema GPS para un lector no especializado. El objetivo es que el lector tenga una idea de todo lo que sucede cuando utiliza algún equipo o aplicación basado en esta tecnología.

\section{GPS, GNSS, Satélites}

Citation: MEDINA-CASTRO, Paul, CARAVEO-MENA, Camilo, BARBOZA-TELLO, Norma Alicia and LOREDOMEDINA, Raúl. A brief description of the GPS architecture. Journal - Democratic Republic of Congo. 2020. 6-11:12-16.

\footnotetext{
* Correspondence to Author (Email: pmedina@uabc.edu.mx)

$\dagger$ Researcher contributing as first author.
} 


\section{Introduction}

The Global Positioning System (GPS) is a navigation system initially developed by the US Department of Defense, and now managed by the US Air Force (U.S. Government, n.d.). The GPS idea was born at the beginning of 70's, and the whole system was completed in 1993, however it was not available for civil use until 1995. Since then, the GPS users have grown enormously around the globe, especially after the mobile's phones were provided with a GPS receiver. The GPS is a radio-satellite system that allows to the GPS receiver to figure out its tridimensional position on the globe (latitude, longitude, and elevation), as well as allows it to determine its velocity and to make precise timing measurements.

Nowadays, GPS is embedded in our daily life, in fact, our actual lifestyle would be unsustainable without GPS technology. The best-known applications of GPS are the locationbased services. In this category are the vehicle or pedestrian navigation applications, which by means of the location on a map give us instructions to reach a destination. Other related applications are: 911 emergency services, children and elderly location, cab service, physical fitness applications, video games, photographs geographic labeling, personalized sales ads, etc. (Park \& Kim, 2020).

In the specific case agriculture applications, GPS has allowed the development of what is known as precision agriculture (Mondal \& Basu, 2009). This consists of taking samples from the ground, where each sample is labeled with its GPS location. The samples are analyzed in the laboratory to obtain information on their nitrogen and organic substance content. With this information is designed a tailored plan to treat the plot with the aim of optimizing resources such as fertilizers, irrigation, pest treatment, etc.

The GPS system is divided in three segments: the space segment, the control segment, and the user segment (U.S. Department of Homeland Security, n.d.). In the next sections of this document is explained how those segments works and how the Assisted-GPS significantly improves the accuracy and speed of the receiver measurements. After that, in the last section are reviewed additional positioning systems.

\section{The Spatial Segment}

The system requires at least 24 Medium Earth Orbit (MEO) satellites, at the date there are 7 additional backup satellites. The satellites height is around 20,200 km above the sea level, it gives them an orbital period of half a day, which guarantees the same satellites positions happening exactly at the same time each day. The satellites are carried to the space by a rocket and released on its orbit at $14,000 \mathrm{~km} / \mathrm{h}$ (ElRabbany, 2002). At this height and velocity, the satellites keep rotating on their orbit due to the equilibrium between the centrifugal force and gravity. The satellites do not require any additional source of energy for orbiting the earth, rather they require energy for the radio communication with the GPS ground stations, and for sending information to the GPS receivers. This energy is provided by batteries recharged by solar panels. The satellites distribution in their orbits guarantees that every spot-on earth is in the coverage area of at least 4 satellites.

The information is transmitted through two radio links: 1) the military band uses a radio carrier of $1,227.60 \mathrm{MHz}$, this signal is encrypted, available for the use of the US government only; 2) the civilian band uses a carrier of $1,575.42$ $\mathrm{MHz}$, available free of charge for every one with a GPS receiver. The signal transmitted power is just $27 \mathrm{~W}$, reaching the earth surface with the extremely low power of $1.41 \times 10-16 \mathrm{~W}$, but through the use of modern techniques of coding, modulation, and signal processing the users on earth can still decode de information sent by satellites (El-Rabbany, 2002).

The information, also known as ephemerids, basically consists in the satellite position and the time of the week, both sampled at the start of transmission. The ephemerids data are 1500 bits transmitted continuously, one after another, at the very slow rate of 50 bits per second. Then the ephemerids transmission last 30 seconds. For the time measurements and as the reference for the digital circuits, the satellite uses an atomic clock, which is the most precise instrument for time measurement, it has an uncertainty of $9 \mathrm{~ns}$. 
The satellite radio signal is encoded by a digital sequence known as PRN (Pseudorandon Noise). Each satellite uses a unique PRN sequence that is used as the satellite identifier. The use of the PRN sequence also reduces the interference between the satellite signals and other communication systems, as well it eases and improves the synchronization between the satellite and the GPS receiver.

\section{The Control Segment}

The control segment consists of a terrestrial network that monitors the transmission of the ephemerids, analyzes the status of each satellite, and sends them synchronization and control data. A different radio link is used for the communication between the ground stations and the satellites than the one used for the transmission of the ephemeris. The ground network has a master station located in the state of Colorado, a backup master station in California, 11 command and control antennas, and 16 monitoring sites located around the globe (U.S. Government, n.d.).

The satellites are precisely tracked by ground stations whose position is precisely known; therefore, the ground network can determine the precise position of each satellite and can even send commands to the satellite to correct it, if necessary. Backup satellites can also be activated or deactivated from the terrestrial network.

It is well known that GPS satellites send weather information to ground stations, and for sure they send other kind of information for Air Force use only.

\section{The user segment}

The signal from each satellite travels towards the earth at the speed of light, and it takes about 77 ms to reach the earth's surface from the satellite. The GPS receiver on earth decodes the place and time the signal was transmitted, and then calculates the time it took for the signal to travel from the satellite to the receiver. In addition, the GPS receiver improves the accuracy of this measurement by observing the phase of the PRN code. With a standard electronic receiver, the measurement of the satellite signal delay can be measured with an accuracy of up to $10 \mathrm{~ns}$.
The GPS receiver measures the signals' delay from at least four satellites. With these measurements, the receiver estimates the distance to each satellite, and since it knows the exact location of each one of them it can estimate its own position. The algorithm that runs the GPS receiver to determine its position is known as "triangulation" or "trilateration", since 3 references are sufficient to determine the position in a three-dimensional space. However, in the GPS system the fourth reference is required to reduce the uncertainty in the delays measurement, otherwise it would be required that each GPS receiver had an atomic clock, which is totally impractical and not feasible (U.S. Department of Homeland Security, n.d.).

\section{Accuracy}

As time is the measured variable, the accuracy of this measurement will determine the accuracy of the receiver position. Like already mentioned, a standard electronic receiver can measure the signal delay with an accuracy of up to $10 \mathrm{~ns}$, which translates into a $3 \mathrm{~m}$ error in the estimation of the distance to the satellite. Thus, a standard receiver has, in theory, an error of $3 \mathrm{~m}$ in estimating its location.

Ideally, if it was possible that the GPS receiver to use an atomic clock, then it will be achieved an accuracy of $1 \mathrm{~ns}$, which is equivalent to an error of $30 \mathrm{~cm}$. This accuracy improvement is too expensive, so techniques have been developed to improve performance without highly increasing the cost of the GPS receiver. For example, military equipment receivers have access to the second GPS channel that allows them to reduce the delay measurement to $1 \mathrm{~ns}$, achieving an uncertainty of $30 \mathrm{~cm}$, without the use of atomic clocks.

The $3 \mathrm{~m}$ accuracy above mentioned is the ideal case, where the unique source of error is time uncertainty, but obviously there are more error sources. One of them is due to the propagation through the ionosphere and the troposphere, which introduce a random delay depending on the environmental conditions and the inclination. Another important source of random error is multipath propagation. It is estimated that, by accumulating the errors of all possible sources, the best accuracy can be up to 15 m (Kaplan \& Hegarty, 2017). 


\section{Assisted-GPS}

The time that a GPS receiver takes to synchronize with only one satellite is in between one and two seconds. Once synchronized, the satellite data decoding last at least 30 seconds, this due to the very slow transmission rate. Thus, it takes about one minute for a GPS receiver to determine its location when it starts working. After this phase, the receiver can update its position every second. In case of communication failure or if the receiver becomes out of sync, which is highly probable, another minute must elapse before the GPS receiver can update its position. This is a big problem, especially in navigation applications, for example, during the landing of an aircraft in a rainy or foggy environment.

The GPS satellites transmit exceptionally low power signals because it was designed for outdoor receivers, with line of sight to the satellites. However, due to the reduction in size and cost of the receivers, and their eminent proliferation, they are now used in places covered by vegetation, surrounded by different constructions, and inside buildings. These users observe a reduction of between 100 and 1000 times in the signal level, making it difficult to obtain information from satellites.

In Assisted-GPS, there are fixed stations which get continuous information from the satellites within their coverage area. This information is made available to nearby GPS users by using an alternative data channel of higher speed and robustness, which is usually another radio link. The GPS receivers instead of decoding the information sent by the satellites, use the information obtained from the fixed stations, so they only need to synchronize with the satellite PRN sequence to improve the estimation of their delay. The synchronization with the PRN sequence is very fast and can be achieved even with very reduced powers. Furthermore, with Assisted-GPS technique, GPS receivers can determine their location in less than a second, even if they have lost communication with the GPS satellites (Kaplan \& Hegarty, 2017).
In addition, the fixed station knows its exact location, and makes a comparison it with the one obtained with their own GPS receiver, obtaining the error of the measurement. The fixed station then sends the estimated error to the mobile receivers, who use this information in order to make the correction, thus decreasing significantly the error. It is known that the Assisted-GPS techniques can achieve accuracies of less than $10 \mathrm{~cm}$ and connection time of less than a second. There are companies dedicated to providing the Assisted-GPS service, some of them even use geostationary satellites. In the case of cellular phones, base stations oversee provide this service, in fact, since the creation of $3 \mathrm{G}$ networks, Assisted-GPS was included as part of any cellular communication standard.

\section{More Positioning Systems}

GPS it is the most popular positioning system currently in operation but is not the only one. There is a Russian option called GLONASS (Global Navigation Satellite System). GLONASS initial development was parallel to that of GPS, but it was delayed by the Soviet Union collapse in 1991. GLONASS also consists of 24 satellites in orbit plus 6 backup satellites, all of them in MEO orbit at 19,100 km high (Information and Analysis Center, 2020). The Russian system is in full operation since 2011 and is included in many devices along with GPS.

China also has its own positioning system, this is called BeiDou, which is the Chinese name for the constellation we know as Ursa Major. This is a hybrid orbit satellite system, 5 GEO and 30 non-GEO (China Satellite Navigation Office, 2020). The first stage of BeiDou began operations in 2000 as a local system, serving only China and its neighbors. It is currently in operation at full capacity with worldwide coverage.

The European Union system is called Galileo. It began providing services in 2016 with half of its satellite system. It was expected that the system would be complete by the end of 2020 , but this has not yet happened. Unlike the other systems, Galileo is of civil creation and management (European GNSS Agency, 2020). 
This system will have a free service with features like those of GPS and GLONASS, and a paid service with much higher accuracy. The four systems are referred to under the general name of GNSS (Global Navigation Satellite System).

\section{Conclusions}

The ancient navigators used stars to locate themselves into the ocean, GPS imitates this idea, so its concept is not novel at all. Science and technology had to go a long way before mankind was able to put its own "guide stars" in the sky, and to build the "sharp eyes" that could observe them. GPS is undoubtedly one of the greatest achievements of modern engineering, which fortunately for users already has competitors. GPS was initially created for vehicle navigation and positioning, but its applications have diversified to include agriculture and entertainment, and more will surely add, as the possibilities of this technology are extensive.

\section{References}

China Satellite Navigation Office. (2020). BeiDou Navigation Satellite System. Retrieved 10 20, 2020, from http://en.beidou.gov.cn/

El-Rabbany, A. (2002). Introduction to GPS: The Global Positioning System. Artech House, Inc. Retrieved 10 2020, from https://www.gps.gov/

European GNSS Agency. (2020). What is Galileo? Retrieved 10 20, 2020, from https://www.gsc-europa.eu/galileo/system

Information and Analysis Center. (2020). Information and analysis center for posittioning, navigation and timing. Retrieved 10 20, 2020, from http://en.beidou.gov.cn

Kaplan, E., \& Hegarty, C. (2017). Understanding GPS/GNSS: Principles and Applications (Third edition ed.). Artech House.

Mondal, P., \& Basu, M. (2009). Adoption of precision agriculture technologies in India and in some developing countries: Scope, present status and strategies. Progress in Natural Science, 19(6), 659-666.
Park, B., \& Kim, S. (2020). An effective digitized GPS signal transmission for high temporal precision IoT services. Journal of Supercomputing, 76(3), 1365-1382.

U.S. Department of Homeland Security. (n.d.). Navigation Center. Retrieved 10 20, 2020, from https://www.navcen.uscg.gov

U.S. Government. (n.d.). GPS.gov. Retrieved 10 20, 2020, from https://www.gps.gov 